\title{
Éditorial
}

\section{Nouvelles avancées dans le domaine des matériaux composites}

Les matériaux composites sont utilisés de façon croissante dans l'industrie aérospatiale et automobile dans un objectif d'allègement des structures associé à des performances élevées. D'autres domaines comme le génie civil et le sport et loisirs constituent également des domaines d'application des matériaux composites. Grace à l'association de leur grande rigidité, grande résistance et faible densité, les matériaux composites permettent de nouvelles conceptions. Néanmoins le potentiel de ces matériaux est lié à des comportements physiques et mécaniques complexes. L'activité de recherche industrielle et académique dans le domaine des matériaux composites est importante. Elle doit déterminer, pour ces matériaux complexes, des lois de comportement, des essais de caractérisation et des méthodes de simulation qui permettent de tirer parti des potentiels importants de ces matériaux. Les travaux présentés dans ce numéro de la revue Matériaux et Techniques sont des recherches réalisées récemment par certains membres de l'Association des Matériaux Composites (AMAC). Ces articles de recherche ont été rédigés à l'issue des Journées Nationales de Composites qui se sont déroulées à Lyon en Juin 2015.

Deux articles présentent des travaux expérimentaux (Argou et al. [1]; Hurmane et al. [2]). Le premier concerne les essais multiaxiaux pour des assemblages composites collés. Le second étend le sujet de l'analyse au dialogue essais/calculs pour des compressions de composites tissés interlocks.

Plusieurs travaux ont pour objectifs la modélisation de différentes facettes du comportement mécanique des matériaux composites. (Eyer et al. [3]) et (Merotte et al. [4]) analysent respectivement l'influence du cisaillement et du taux de porosité. (Foti et al. [5]) présentent une étude de fatigue multi-physique et (Krasnobrizha et al. [6]) étudient le comportement d'hystérésis des matériaux composites.

Trois articles concernent des études de simulations numériques et de modélisation des matériaux composites. (Girardot et al. [7]) modélisent des impacts sur des renforts tissés. (Naouar et al. [8]) utilisent la tomographie X pour des simulations mesoscopiques. (Perret et al. [9]) modélisent les instabilités de panneaux composites.

Deux papiers concernent la simulation des procédés composites. (Sassi et al. [10]) analysent la caractérisation électrique d'un CRFP en cours de cuisson. (Blais et al. [11]) présentent une méthode de simulation numérique du procédé d'infusion.

\section{Références}

[1] N. Argoud, J. Rousseau, B. Piezel, A. Fiore, S. Fontaine, Matériaux \& Techniques 104 (2016) 402

[2] A. Hurmane, F.-X. Irisarri, F. Laurin, S. Leclercq, Malk Benzeggagh, Matériaux \& Techniques 104 (2016) 403

[3] G. Eyer, O. Montagnier, J.-P. Charles, F. Mazerolle, C. Hochard, Matériaux \& Techniques 104 (2016) 404

[4] J. Merotte, A. Le Duigou, A. Bourmaud, K. Behlouli, C. Baley, Matériaux \& Techniques 104 (2016) 405

[5] F. Foti, Y. Pannier, M. Gigliotti, M.C. Lafarie-Frenot, D. Mellier, Thach Can Luu, Matériaux \& Techniques 104 (2016) 406

[6] A. Krasnobrizha, P. Rozycki, P. Cosson, L. Gorneta, Matériaux \& Techniques 104 (2016) 407

[7] J. Girardot, F. Dau, Matériaux \& Techniques 104 (2016) 408

[8] N. Naouar, E. Vidal-Salle, E. Maire, J. Schneider, P. Boisse, Matériaux \& Techniques 104 (2016) 409

[9] O. Perret, A. Lebée, C. Douthe, K. Sab, Matériaux \& Techniques 104 (2016) 410

[10] S. Sassi, P. Marguerès, R. Le Begue, M. Mounkaila, P. Olivier, T. Camps, Matériaux \& Techniques 104 (2016) 411

[11] M. Blais, N. Moulin, P.-J. Liotier, S. Drapier, Matériaux \& Techniques 104 (2016) 412 